BULL. AUSTRAL. MATH. SOC.

VOL. $4(197 \mid), 287$.

\title{
The stability of massive hydrogen stars near the main sequence
}

\author{
S.M.A. Meggitt
}

An astrophysical problem of great interest is that of establishing theoretical mass limits for stars. In this thesis an investigation is made into the maximum stable mass for stars formed from pure hydrogen.

Initial models of pure hydrogen stars are constructed numerically for a number of masses, these models being in a state of steady homologous contraction, with an energy source which is entirely gravitational. Henyey's method is then used to follow the evolution of these models during the appearance of nuclear reactions, the contraction to a minimum radius, and the subsequent expansion to a steady main-sequence state. The small amounts of helium and carbon produced have a large effect on the radii of the models, and it is the continued formation of these elements which causes the main-sequence radii to be substantially greater than the minimum radii. Stability against radial oscillations is tested at each step. The stability is found to decrease as nuclear energy increases in importance, the period of greatest instability occuring shortly after minimum radius.

It is found that stars with masses greater than 200 solar masses become pulsationally unstable before their contraction is complete, while stars of lower mass can reach the main-sequence and continue their evolution beyond it.

Received 4 December 1970. Thesis submitted to the Australian National University, January 1969. Degree approved, November 1969. Supervisors: Professor R.F.E. Van der Borght, Dr I.T. Searle, Professor A. Brown. 